\title{
Fat-soluble vitamins and plasma and erythrocyte membrane fatty acids in chylothorax pediatric patients receiving a medium-chain triglyceride-rich diet
}

\author{
Narumon Densupsoontorn, ${ }^{1, *}$ Pipop Jirapinyo, ${ }^{1}$ Hathaichanok Tirapongporn, ${ }^{1}$ Renu Wongarn, ${ }^{1}$ \\ Kwanjai Chotipanang, ${ }^{1}$ Phakkanan Phuangphan ${ }^{1}$ and Nalinee Chongviriyaphan ${ }^{2}$
}

1'Department of Pediatrics, Faculty of Medicine Siriraj Hospital, Mahidol University, Bangkok, 10700, Thailand

${ }^{2}$ Department of Pediatrics, Faculty of Medicine Ramathibodi Hospital, Mahidol University, Bangkok, 10400, Thailand

(Received 28 February, 2014; Accepted 25 May, 2014; Published online 4 October, 2014)

\begin{abstract}
Post-operative chylothorax can be cured by a medium-chain triglyceride (MCT)-rich diet. However, there is concern that an MCT-rich diet results in clinical and biochemical deficiencies in fat-soluble vitamins and fatty acids. We compared fat-soluble vitamins status and fatty acids status before and after administration of an MCT-rich diet. Nine children with congenital heart disease developed chylothorax after cardiac surgery. Blood samples were drawn from each subject twice, first prior to administration of an MCT-rich diet and secondly when the chylothorax was clinically cured and the MCT diet discontinued. Both blood samples were analyzed for retinol and 25-hydroxy vitamin D concentrations, the ratio of $\alpha$-tocopherol to total lipids $(\alpha-T E / T L)$, coagulogram, and the fatty acid composition in plasma and erythrocyte membrane phospholipids. In spite of a decrease in the $\alpha$-TE/TL ratio ( $3.78 \pm 0.89$ vs $2.36 \pm 0.44 \mathrm{mg} / \mathrm{g}, p<0.05)$, this decrease did not reach the deficiency cut-off level. Linoleic acid in both plasma and erythrocyte membrane lipids decreased significantly $(25.25 \pm 8.06$ vs $14.25 \pm 2.88 \%$, and $11.19 \pm 2.15$ vs $6.89 \pm 2.45 \%$, respectively). Administration of an MCT-rich diet for treatment of postoperative chylothorax caused a reduction in vitamin $\mathrm{E}$ status and linoleic acid, but without any symptoms of deficiency.
\end{abstract}

Key Words: chylothorax, fat-soluble vitamins, fatty acids, MCT-rich diet hylothorax is a condition of chyle leakage from lymphatic
vessels into the pleural cavity. Post-intrathoracic surgery is
the main cause of chylothorax in children with one study reporting
an incidence of $0.89 \%^{(1-3)}$ The same study suggested that most
cases of post-operative chylothorax can be cured by a medium-
chain triglyceride (MCT)-rich diet. ${ }^{(3)}$
The essential fatty acids, linoleic acid and $\alpha$-linolenic acid, are
long-chain polyunsaturated fatty acids that cannot be synthesized
by humans because of the absence of the $\Delta^{12}$ and $\Delta^{15}$ enzymes
(counting from the carboxyl terminal) necessary to insert a double
bond at the $\omega-6$ and $\omega-3$ positions of a fatty acid carbon chain. The
essential fatty acids must therefore be supplied in the diet. ${ }^{(4)}$ Most
dietary triglycerides (TGs) and fat-soluble vitamins are incorpo-
rated into the chylomicrons for absorption into the lymphatic
system. Intestinal absorption of fat-soluble vitamins is reduced by
too little dietary fat or a lack of bile salts. Fatty acids with chain
lengths of less than 12 carbon atoms are also absorbed passively
across the intestinal brush-border membrane and transported
through the portal vein, (5) whereas fatty acids with chain lengths of
more than 12 carbon atoms and cholesterol have a rate-limiting step when passing across the unstirred water layer of enterocytes and transported through the lymphatic vessels. ${ }^{(6)}$ Therefore MCT oil containing fatty acids with chain lengths of 8-10 carbon atoms esterified to glycerol, substituted for long-chain triglyceride (LCT) oil, is therapeutically used for primary effective treatment of post-operative chylothorax or chylopericardium. (3)

In post-operative chylothorax patients, there is concern that an MCT-rich diet will result in clinical and biochemical deficiencies in fat-soluble vitamins and fatty acids. The effects of an MCT-rich diet used for management of post-operative chylothorax on fatsoluble vitamins status and fatty acids status are not known. The aims of this study were to compare levels of fat-soluble vitamins and fatty acids in pediatric patients with post-operative chylothorax before and after treatment with an MCT-rich diet.

\section{Materials and Methods}

The study was approved by the Siriraj Institutional Review Board. The parents of all subjects gave their informed consent for their children to be included in the study.

Study design. The study was a before-after study design with the same subjects.

Study subjects. In a previous study, ${ }^{(3)}$ the authors reported the incidence of post-operative chylothorax was $0.89 \%$. The minimum required sample size for this study, calculated from the pilot study with a mean difference of linoleic acid $6.84 \%$ and a standard deviation of the difference $6.77 \%, \alpha$-error $5 \%$, and $\beta$ error $20 \%$, was determined to be eight subjects, who were then recruited from November 2008 until September 2013.

The study included children who developed chylothorax or chylopericardium after undergoing a cardiothoracic operation, for which nutritional treatment with an MCT-rich diet was prescribed. Inclusion criteria were (i) appropriate-to-age preoperative dietary intake, and (ii) postoperative fluid accumulation in either the pleural cavity or pericardial cavity proven to be chylous fluid, defined as milky sterile fluid, TG concentration $>110 \mathrm{mg} / \mathrm{dl}$ and higher than serum TG concentration, and lymphocytic predominance. ${ }^{(7)}$ Exclusion criteria were the administration of intravenous lipid emulsion and/or vitamin supplementation either prior to the diagnosis or during their treatment for chylothorax. Eleven subjects met the initial enrolment criteria of postoperative chylothorax, and nine subjects were recruited to the study.

*To whom correspondence should be addressed. E-mail: narumon.den@mahidol.ac.th 
Table 1. Characteristics of nine subjects with chylothorax treated with an MCT-rich diet

\begin{tabular}{|c|c|c|c|c|c|c|c|c|c|c|}
\hline $\begin{array}{l}\text { Subject } \\
\text { No. }\end{array}$ & Sex & Age & $\begin{array}{l}\text { BW } \\
(\mathrm{kg})\end{array}$ & $\begin{array}{l}\text { Lt or } \mathrm{Ht} \\
(\mathrm{cm})\end{array}$ & $\% W A$ & $\begin{array}{l}\text { \%LA or } \\
\% \text { HA }\end{array}$ & $\begin{array}{l}\text { \%WL or } \\
\% \text { WH }\end{array}$ & $\begin{array}{l}\text { Presence of chylothorax } \\
\text { and/or chylopericardium }\end{array}$ & $\begin{array}{c}\text { Duration of } \\
\text { MCT-rich diet (days) }\end{array}$ & Result \\
\hline 1 & $\mathrm{~F}$ & 15 years & 27 & 138 & 58.7 & 88.46 & 88.52 & R chylothorax & 27 & Cured \\
\hline 2 & $M$ & 11.5 months & 6.5 & 64 & 69.89 & 85.33 & 95.59 & R chylothorax & 28 & Cured \\
\hline 3 & $\mathrm{M}$ & 1 month & 2.7 & 46 & 65.85 & 84.4 & 100 & R chylothorax & 29 & Cured \\
\hline 4 & $\mathrm{M}$ & 5.8 years & 25.1 & 122 & 128.72 & 107.96 & 111.56 & L chylothorax & 31 & Cured \\
\hline 5 & $\mathrm{M}$ & 8 months & 5.1 & 65 & 62.2 & 92.86 & 72.86 & L chylothorax & 28 & Cured \\
\hline 6 & $\mathrm{M}$ & 12 months & 7 & 70 & 74.47 & 92.72 & 85.37 & R chylothorax & 28 & Cured \\
\hline 7 & $\mathrm{~F}$ & 8.2 years & 25.1 & 115 & 109.13 & 92.37 & 128.72 & B chylothorax & 28 & Cured \\
\hline 8 & $\mathrm{M}$ & 21.6 months & 6.98 & 74 & 59.15 & 87.57 & 76.7 & R chylothorax & 29 & Cured \\
\hline 9 & $\mathrm{M}$ & 2.7 years & 12 & 89 & 85.71 & 95.7 & 93.02 & R chylothorax & 27 & Cured \\
\hline
\end{tabular}

MCT; medium-chain triglyceride, BW; body weight, Lt; length, Ht; height, \%LA; \%length-for-age, \%HA; \%height-for-age, \%WL; \%Weight-forlength, \%WH; \%weight-for-height, F; female, M; male, R; right, L; left, B; bilateral.

Study procedure. Blood samples were drawn from the subjects twice, the first prior to initiation of the MCT-rich diet and the second after the chylous leakage was clinically determined to be cured and the MCT-rich diet discontinued. The MCT-rich diet was composed of non-fat milk, semisolid, or solid foods supplemented with MCT oil depending on the age of the child.

Blood sampling and analysis. All blood samples were analyzed for (i) fat-soluble vitamins, which were vitamin A (retinol), 25 -hydroxy vitamin D (25-OHD), vitamin E ( $\alpha$-tocopherol), partial thromboplastin time (PTT), and prothrombin time (PT), (ii) TG and cholesterol, and (iii) plasma phospholipids and erythrocyte membrane phospholipids fatty acids.

The blood samples were placed in an EDTA-containing tube and centrifuged to collect the plasma and red blood cells, which were then stored at $-20^{\circ} \mathrm{C}$ until analysis. All procedures for analyzing vitamins $\mathrm{A}$ and $\mathrm{E}$ were performed under light protection. Concentrations of retinol and $\alpha$-tocopherol were measured by using high performance liquid chromatography as previously described. ${ }^{(8,9)}$ A plasma retinol concentration of $<20 \mathrm{mcg} / \mathrm{dl}$ and a ratio of $\alpha$-tocopherol to total lipids ( $\alpha$-TE/total lipids) of $<0.8 \mathrm{mg} / \mathrm{g}$ were considered to indicate deficiency. Analysis of 25-OHD was performed using an electrochemiluminescence immunoassay (Roche Diagnostics, IN). A serum 25-OHD concentration of $<20 \mathrm{ng} / \mathrm{dl}$ was considered to indicate deficiency. ${ }^{(10,11)}$ Partial thromboplastin and prothrombin times were determined using the Dade Actin FS Activated PTT reagent and Thromborel S reagent, respectively (Siemens Healthcare Diagnostic Inc., Newark, DE). Cut-off values were derived from age-dependent references. ${ }^{(12)}$ Plasma concentrations of TG and cholesterol were measured using an enzymatic colorimetric method (Roche Diagnostics, Indianapolis, IN).

The analysis of fatty acids was performed using a gas-liquid chromatography method as described elsewhere. ${ }^{(13,14)}$ The kind and amount of individual fatty acids in the plasma phospholipids and erythrocyte membrane phospholipids were calculated and the results were expressed as percentages of total fatty acids with chain lengths of 10 and 24 (C10-C24) in each sample.

Paired blood samples were analyzed for vitamin status in all nine subjects, but measured for fatty acids in only seven subjects due to incomplete blood tests.

Statistical analysis. Statistical analysis was performed with SPSS for Windows version 15.0. Data concerning fat-soluble vitamins (retinol, 25-OHD, tocopherol, PTT, and PT) were categorized into deficient/normal groups or abnormally prolonged/ normal groups according to the cut-off values, and compared by the McNemar test. Normally distributed raw data, such as retinol, 25-OHD, tocopherol levels, PTT, PT, and fatty acid levels, before and after treatment with the MCT diet, were shown as mean values and standard deviations, and then compared by paired $t$ test. A $p$ value less than 0.05 was deemed to be statistically significant.

\section{Results}

In total, seven male and two female patients were recruited with a mean age of 4 years (SD 4.92 years, min-max 1 month-15 years). All subjects presented with post-operative chylothorax (6: right side, 2: left side, and 1: bilateral) in the absence of chylopericardium (Table 1). Chylothorax was completely cured in all subjects and no chylous leakage reappeared in any patients after reintroduction of the normal diet.

At baseline, most of the subjects were below average in three growth parameters, \%weight-for-age ( 7 out of 9 ), \%length-for-age or \%height-for-age ( 7 out of 9 ), and \%weight-for-length or \%weight-for-height (4 out of 9) (Table 1). Initial baseline biochemical indexes found vitamin A deficiency in 3 of 9, vitamin D deficiency in 4 of 9 , and vitamin $\mathrm{K}$ deficiency without clinical bleeding in 6 of 8 patients by minimally prolonged PT with an average difference \pm SD from upper normal limit of $2.37 \pm 1.52 \mathrm{~s}$, but normal PTT. None had vitamin E deficiency. Warfarin, a vitamin $\mathrm{K}$-antagonist, was prescribed to subject 8 before initiation of the MCT diet to treat a clot in the Glen shunt connecting the superior vena cava and the right pulmonary artery, and therefore, his blood was not analyzed for PTT and PT. Malnutrition, defined by all three growth parameters of the patients in this study, was not significantly associated with deficiencies in fat-soluble vitamins.

After treatment with the MCT diet, retinol and 25-OHD concentrations, the ratios of $\alpha$-TE:total lipids, PTTs, and PTs were not statistically significantly different from the baseline data according to the cut-off values (Table 2). Meanwhile, the mean retinol concentration increased from 33.73 to $45.07 \mathrm{mg} / \mathrm{dl}$ $(p<0.05)$ and the mean ratio of $\alpha$-TE:total lipids was decreased significantly compared to the baseline data $(3.78 \mathrm{vs} 2.36 \mathrm{mg} / \mathrm{g}$, $p<0.05)$, even though they were still within the normal ranges.

Regarding plasma and erythrocyte membrane lipids, only linoleic acid decreased significantly after treatment with the MCT diet compared to the baseline concentrations $(25.25 \pm 8.06 \%$ vs $14.25 \pm 2.88 \% ; p<0.05$, and $11.19 \pm 2.15 \%$ vs $6.89 \pm 2.45 \%$; $p<0.05$, respectively, $n=7$ ), whereas the before-after concentrations of $\alpha$-linolenic acid, oleic acid, arachidonic acid, eicosapentaenoic acid and docosahexaenoic acid were not statistically different (Tables 3 and 4).

\section{Discussion}

This study found that the mean ratio of $\alpha$-tocopherol to total lipids and the values of linoleic acid in both plasma and erythrocyte membrane lipids decreased significantly after a 28-day MCTrich diet treatment for post-operative chylothorax compared to initial values.

The rationale of managing post-operative chylothorax is to restrict the intake of long-chain fatty acids (LCFAs) from the diet in order to decrease lymphatic flow through leakage points 
Table 2. Biochemical index of fat-soluble vitamins before and after treatment of chylothorax with an MCT-rich diet

\begin{tabular}{|c|c|c|c|c|c|c|c|c|c|c|c|c|}
\hline \multirow{2}{*}{$\begin{array}{c}\text { Subject } \\
\text { No. }\end{array}$} & \multicolumn{2}{|c|}{ Retinol $^{\ddagger}$ (mcg/dl) } & \multicolumn{2}{|c|}{$25-\mathrm{OHD}^{\ddagger}(\mathrm{ng} / \mathrm{ml})$} & \multicolumn{2}{|c|}{$\alpha-\mathrm{TE}^{\ddagger}(\mu \mathrm{g} / \mathrm{dl})$} & \multicolumn{2}{|c|}{$\alpha$-TE/total lipids ${ }^{\ddagger}(\mathrm{mg} / \mathrm{g})$} & \multicolumn{2}{|c|}{ PTT $^{\ddagger}$} & \multicolumn{2}{|c|}{$\mathrm{PT}^{\ddagger}$} \\
\hline & Before & After & Before & After & Before & After & Before & After & Before & After & Before & After \\
\hline 1 & 15.87 & 11.19 & 6.69 & 16.45 & 1366.29 & 684.75 & NA & 4.28 & 24.70 & 20.40 & 14.10 & 14.60 \\
\hline 2 & 29.99 & 33.88 & 25.35 & 34.47 & 1188.68 & 611.84 & 4.40 & 2.44 & 22.70 & 24.70 & 11.60 & 11.70 \\
\hline 3 & 25.97 & 38.21 & 24.04 & 8.17 & 1091.10 & 422.27 & 5.01 & 2.02 & 26.60 & 31.30 & 13.50 & 13.70 \\
\hline 4 & 46.36 & 43.84 & 22.70 & 23.20 & 629.08 & 616.30 & 2.35 & 3.16 & 27.30 & 28.00 & 14.50 & 14.30 \\
\hline 5 & 45.70 & 66.20 & 26.20 & 23.20 & 1036.17 & 423.08 & 4.26 & 2.03 & 25.10 & 25.90 & 10.90 & 11.70 \\
\hline 6 & 15.39 & 47.80 & 7.29 & 10.40 & 1458.38 & 770.42 & 3.75 & 1.75 & 25.60 & 39.00 & 16.20 & 22.10 \\
\hline 7 & 37.61 & 69.58 & 11.90 & 4.64 & 494.67 & 619.13 & 2.65 & 2.63 & 24.20 & 24.20 & 14.20 & 13.90 \\
\hline 8 & 68.46 & 65.95 & 29.18 & 26.21 & 767.38 & 418.00 & 3.97 & 2.53 & NA & NA & NA & NA \\
\hline 9 & 18.23 & 28.97 & 9.94 & 14.02 & 845.73 & 590.23 & 3.90 & 2.35 & 23.60 & 26.90 & 13.20 & 13.10 \\
\hline Mean \pm SD & $33.73 \pm 17.65$ & $45.07 \pm 19.59$ & $18.14 \pm 9.01$ & $17.86 \pm 9.64$ & $986.39 \pm 327.62$ & $572.89 \pm 125.74$ & $3.78 \pm 0.89$ & $2.36 \pm 0.44$ & $24.97 \pm 1.52$ & $27.55 \pm 5.59$ & $13.52 \pm 1.67$ & $14.39 \pm 3.30$ \\
\hline
\end{tabular}

F; female, M; male, NA; not available, 25-OHD; 25-hydroxy vitamin D, $\alpha$-TE; $\alpha$-tocopherol, $\alpha$-TE/total lipids; $\alpha$-tocopherol/sum of cholesterol and triglyceride, PTT; partial thromboplastin time, PT; prothrombin time, NA; not available. ${ }^{\ddagger}$ McNemar test used for comparing data between before and after 28 days of a MCT-rich diet, categorized as deficient/ normal groups or abnormally prolonged/normal groups according to the cut-off values.

Table 3. Plasma phospholipid fatty acids before and after treatment of chylothorax with an MCT-rich diet ${ }^{\dagger}$

\begin{tabular}{|c|c|c|c|c|c|c|c|c|c|c|c|c|}
\hline \multirow{2}{*}{$\begin{array}{c}\text { Subject } \\
\text { No. }\end{array}$} & \multicolumn{2}{|c|}{$\mathrm{LA}^{\ddagger, *}$} & \multicolumn{2}{|c|}{$\mathrm{ALA}^{\ddagger}$} & \multicolumn{2}{|c|}{$\mathrm{OA}^{\ddagger}$} & \multicolumn{2}{|c|}{ ARA $^{\ddagger}$} & \multicolumn{2}{|c|}{$\mathrm{EPA}^{\ddagger}$} & \multicolumn{2}{|c|}{$\mathrm{DHA}^{\ddagger}$} \\
\hline & Before & After & Before & After & Before & After & Before & After & Before & After & Before & After \\
\hline 1 & NA & NA & NA & NA & NA & NA & NA & NA & NA & NA & NA & NA \\
\hline 2 & NA & NA & NA & NA & NA & NA & NA & NA & NA & NA & NA & NA \\
\hline 3 & 24.5 & 16.00 & 0 & 0.60 & 28.35 & 36.39 & 5.82 & 3.05 & 0 & 0.24 & 0.59 & 1.25 \\
\hline 4 & 18.71 & 16.88 & 0 & 0 & 38.16 & 29.77 & 3.08 & 5.58 & 0 & 0 & 1.16 & 1.26 \\
\hline 5 & 28.48 & 9.98 & 0.48 & 0.19 & 29.68 & 36.30 & 3.57 & 4.27 & 0.14 & 0.22 & 1.86 & 0.81 \\
\hline 6 & 35.53 & 10.76 & 0.28 & 0.22 & 24.72 & 36.38 & 4.29 & 2.68 & 0 & 0.16 & 1.48 & 0.47 \\
\hline 7 & 14.74 & 13.77 & 0.24 & 0 & 30.01 & 30.28 & 4.94 & 3.84 & 0.20 & 0.19 & 1.85 & 1.40 \\
\hline 8 & 34.84 & 17.12 & 0.63 & 0.23 & 21.19 & 27.34 & 4.34 & 6.53 & 0.13 & 0.23 & 1.57 & 0.83 \\
\hline 9 & 19.94 & 15.22 & 0.08 & 0.13 & 32.49 & 27.40 & 4.06 & 3.03 & 0.16 & 0.15 & 0.80 & 0.53 \\
\hline Mean \pm SD & $25.25 \pm 8.06$ & $14.25 \pm 2.88$ & $0.24 \pm 0.24$ & $0.17 \pm 0.19$ & $29.23 \pm 5.43$ & $32.12 \pm 3.94$ & $4.30 \pm 0.89$ & $4.15 \pm 1.33$ & $0.09 \pm 0.08$ & $0.15 \pm 0.09$ & $1.33 \pm 0.49$ & $0.82 \pm 0.47$ \\
\hline
\end{tabular}

${ }^{\dagger}$ Individual fatty acids presented as percentage of total fatty acids. ${ }^{\ddagger}$ Paired Student $t$ test used for comparing data between before and after a 28 day MCT-rich diet; ${ }^{*} p<0.05$. LA; Linoleic acid, ALA; $\alpha$-Linolenic acid, OA; Oleic acid, ARA; Arachidonic acid, EPA; Eicosapentaenoic acid, DHA; Docosahexaenoic acid, NA; not available.

Table 4. Erythrocyte membrane fatty acids before and after treatment of chylothorax with an MCT-rich diet ${ }^{\dagger}$

\begin{tabular}{|c|c|c|c|c|c|c|c|c|c|c|c|c|}
\hline \multirow{2}{*}{$\begin{array}{c}\text { Subject } \\
\text { No. }\end{array}$} & \multicolumn{2}{|c|}{$\mathrm{LA}^{\ddagger, *}$} & \multicolumn{2}{|c|}{$\mathrm{ALA}^{\ddagger}$} & \multicolumn{2}{|c|}{$O A^{\ddagger}$} & \multicolumn{2}{|c|}{ ARA $^{\ddagger}$} & \multicolumn{2}{|c|}{$\mathrm{EPA}^{\ddagger}$} & \multicolumn{2}{|c|}{$\mathrm{DHA}^{\ddagger}$} \\
\hline & Before & After & Before & After & Before & After & Before & After & Before & After & Before & After \\
\hline 1 & NA & NA & NA & NA & NA & NA & NA & NA & NA & NA & NA & NA \\
\hline 2 & NA & NA & NA & NA & NA & NA & NA & NA & NA & NA & NA & NA \\
\hline 3 & 12.59 & 8.77 & 0 & 0 & 19.96 & 27.65 & 11.51 & 9.37 & 0 & 0 & 1.24 & 3.66 \\
\hline 4 & 11.55 & 7.31 & 0 & 0 & 21.35 & 16.13 & 9.17 & 11.84 & 0 & 0 & 4.31 & 4.17 \\
\hline 5 & 13.98 & 6.01 & 0.19 & 0.34 & 16.01 & 18.59 & 10.61 & 11.76 & 0.30 & 0.36 & 5.25 & 4.42 \\
\hline 6 & 9.88 & 7.62 & 4.06 & 0.10 & 12.20 & 23.53 & 6.64 & 8.81 & 0.16 & 0.35 & 2.28 & 2.67 \\
\hline 7 & 8.36 & 1.77 & 0.09 & 0 & 16.27 & 5.90 & 12.62 & 0.48 & 0.32 & 0 & 5.33 & 0.21 \\
\hline 8 & 13.01 & 8.79 & 0.16 & 0 & 10.92 & 14.39 & 12.44 & 14.75 & 0.19 & 0.26 & 3.70 & 3.49 \\
\hline 9 & 9.00 & 7.99 & 0.08 & 0.09 & 16.37 & 15.72 & 12.94 & 11.70 & 0.41 & 0.38 & 2.66 & 3.15 \\
\hline Mean \pm SD & $11.19 \pm 2.1$ & $6.89 \pm 2.45$ & $0.65 \pm 1.50$ & $0.08 \pm 0.12$ & $16.15 \pm 3.75$ & $17.41 \pm 6.95$ & $10.85 \pm 2.27$ & $9.81 \pm 4.55$ & $0.20 \pm 0.16$ & $0.19 \pm 0.18$ & $3.54 \pm 1.55$ & $3.11 \pm 1.41$ \\
\hline
\end{tabular}

${ }^{\dagger}$ Individual fatty acids presented as percentage of total fatty acids. ${ }^{\ddagger}$ Paired Student $t$ test used for comparing data between before and after treatment with the MCT-rich diet; ${ }^{\star} p<0.05$. LA; Linoleic acid, ALA; $\alpha$-Linolenic acid, OA; Oleic acid, ARA; Arachidonic acid, EPA; Eicosapentaenoic acid, DHA; Docosahexaenoic acid, NA; not available.

because LCFAs are transported via chylomicrons along the lymphatic vessels. To maintain the energy density of this special diet, medium-chain fatty acids in the MCT oil are substituted for the LCFAs. An MCT-rich diet consists of non-fat milk, semisolid, or solid foods supplemented with MCT oil, depending on the patient's age and requirements.

The initial mean retinol concentration of all subjects was normal and it was increased after treatment with the MCT-rich diet, which may be explained by adequate retinol hepatic storage.

Vitamin D deficiency found prior to beginning an MCT-rich diet may reflect a low dietary intake and/or insufficient sunlight exposure from limitation of outdoor exercise due to exhaustion from cardiac disease. Inadequate sunlight exposure is a risk factor for vitamin D deficiency. ${ }^{(15)}$ No significant change in vitamin D concentration after a 28-day dietary restriction, in spite of the low natural-light UVB exposure during hospital admission and no environmental change while living at a patient's home, would seem to indicate that there was no effect of a 28-day MCT-rich diet on vitamin D status.

Although the average vitamin E status, represented as the $\alpha$ tocopherol:total lipids ratio, was reduced significantly, such data did not reach the deficient cut-off value, which likely indicated that the subjects had sufficient storage prior to their surgery.

Vitamin K deficiency, which manifests as clinically significant bleeding, is shown by prolonged PT and PTT. Such values must be interpreted on the basis of the subject's age reference. The present study found that two-thirds of the subjects had minimally prolonged PT in the absence of prolonged PTT at baseline. This 
can be explained by (i) vitamin $\mathrm{K}$ is an essential factor for hepatic gamma-glutamyl carboxylase that adds a carboxyl group to glutamic acid residues on factors II, VII, IX and X. Prothrombin time is used to measure factors I, II, V, VII, and X of the extrinsic pathway of coagulation; and/or (ii) factor VII has the shortest half-life of the coagulation factors. In early vitamin $\mathrm{K}$ deficiency, PPT may be normal or mildly prolonged. In our study, no significant changes in the vitamin $\mathrm{K}$ status of our subjects after their MCT-rich diet may reflect adequate vitamin $\mathrm{K}$ storage prior to the 28-day diet or production by intestinal bacteria.

Linoleic acid values, but not $\alpha$-linolenic acid, in both plasma and erythrocyte membrane decreased significantly after LCTrestriction for 28 days. Linoleic acid and $\alpha$-linolenic acid are essential fatty acids for humans, which are required from dietary supplementation. Long-chain polyunsaturated fatty acids (PUFAs) are indispensable for the growing tissues in children especially the brain and retina, and for synthesizing eicosanoids. The dietary essential fatty acids status is reflected in the pattern of PUFAs in plasma and erythrocyte membranes. ${ }^{(16)}$ The fatty acid composition of erythrocyte membrane lipids better reflects tissue deposition than plasma lipids because of the long half-life of erythrocytes. ${ }^{(17)}$ The daily requirement of dietary $\alpha$-linolenic acid is much less than that of linoleic acid. ${ }^{(18)}$ This, therefore, resulted in a nonsignificant reduction in plasma and erythrocyte membrane $\alpha$ linolenic acid values.

The implications of the present study are as follows: (i) nutritional support through an MCT-rich diet for about 28 days is an effective treatment for post-operative chylothorax, although it may involve statistically significant decreases in linoleic acid and vitamin E. Such reductions are transient and asymptomatic; (ii) an intravenous lipid emulsion with fat-soluble vitamins may be additionally administered to prevent essential fatty acid deficiency, but a risk of catheter-related infection has to be considered;

\section{References}

1 Bond SJ, Guzzetta PC, Snyder ML, Randolph JG. Management of pediatric postoperative chylothorax. Ann Thorac Surg 1993; 56: 469-472.

2 Büttiker V, Fanconi S, Burger R. Chylothorax in children: guidelines for diagnosis and management. Chest 1999; 116: 682-687.

3 Densupsoontorn NS, Jirapinyo P, Wongarn R, et al. Management of chylothorax and chylopericardium in pediatric patients: experiences at Siriraj Hospital, Bangkok. Asia Pac J Clin Nutr 2005; 14: 182-187.

4 Mayes PA. Metabolism of unsaturated fatty acids and eicosanoids. In: Murray RK, Granner DK, Mayes PA, Rodwell VW, eds. Harper's Biochemistry, 25th ed, Stamford: Appleton \& Lange, 2000; 250-258.

5 Bracco U. Effect of triglyceride structure on fat absorption. Am J Clin Nutr 1994; 60 (6 Suppl): 1002S-1009S.

6 Thomson AB, Schoeller C, Keelan M, Smith L, Clandinin MT. Lipid absorption: passing through the unstirred layers, brush-border membrane, and beyond. Can J Physiol Pharmacol 1993; 71: 531-555.

7 Valentine VG, Raffin TA. The management of chylothorax. Chest 1992; 102: $586-591$.

8 Kositamongkol S, Suthutvoravut U, Chongviriyaphan N, Feungpean B, Nuntnarumit P. Vitamin A and E status in very low birth weight infants. $J$ Perinatol 2011; 31: 471-476.

9 Bieri JG, Tolliver TJ, Catignani GL. Simultaneous determination of alphatocopherol and retinol in plasma or red cells by high pressure liquid chromatography. Am J Clin Nutr 1979; 32: 2143-2149.

10 Wagner CL, Greer FR; American Academy of Pediatrics Section on Breast- and (iii) nutritional support and sunlight exposure for children with congenital heart disease before surgery must be provided and encouraged to prevent protein-energy malnutrition and nutrient deficiencies.

In conclusion, administration of a 28-day, MCT-rich diet for treatment of post-operative chylothorax causes reduction in vitamin $\mathrm{E}$ concentrations, but not to a dangerous level. Linoleic acid values also decrease, but again without any significant symptoms.

\section{Acknowledgments}

This study was supported by the Siriraj Research Development Fund and a "Chalermphrakiat" Grant from the Faculty of Medicine Siriraj Hospital, Mahidol University, Thailand.

\section{Abbreviations}

$\alpha$-TE/TL a ratio of $\alpha$-tocopherol to total lipids

EDTA ethylenediaminetetraacetic acid

LCFAs long-chain fatty acids

LCT long-chain triglyceride

MCT medium-chain triglyceride

PTT partial thromboplastin time

PUFAs polyunsaturated fatty acids

PT prothrombin time

TGs triglycerides

25-OHD 25-hydroxy vitamin D

\section{Conflict of Interest}

No potential conflicts of interest were disclosed.

feeding; American Academy of Pediatrics Committee on Nutrition. Prevention of rickets and vitamin D deficiency in infants, children, and adolescents. Pediatrics 2008; 122: 1142-1152.

11 Belenchia AM, Tosh AK, Hillman LS, Peterson CA. Correcting vitamin D insufficiency improves insulin sensitivity in obese adolescents: a randomized controlled trial. Am J Clin Nutr 2013; 97: 774-781.

12 Scott JP, Raffini LJ, Montgomery RR. Hemostasis. In: Kliegman RM, Stanton BF, St. Geme JW, Schor NF, Behrman RE, eds. Nelson Textbook of Pediatrics. 19th ed, Philadelphia: Elsevier Saunders, 2011; 1693-1699.

13 Lepage G, Roy CC. Direct transesterification of all classes of lipids in a one-step reaction. $J$ Lipid Res 1986; 27: 114-120.

14 Jirapinyo P, Densupsoontorn N, Wiraboonchai D, et al. Fatty acid composition in breast milk from 4 regions of Thailand. J Med Assoc Thai 2008; 91: 1833-1837.

15 Casey CF, Slawson DC, Neal LR. VItamin D supplementation in infants, children, and adolescents. Am Fam Physician 2010; 81: 745-748.

16 Holman RT. The ratio of trienoic: tetraenoic acids in tissue lipids as a measure of essential fatty acid requirement. J Nutr 1960; 70: 405-410.

17 Jakobik V, Burus I, Decsi T. Fatty acid composition of erythrocyte membrane lipids in healthy subjects from birth to young adulthood. Eur $J$ Pediatr 2009; 168: 141-147.

18 American Academy of Pediatrics. Fat and fatty acids. In: Kleinman RE, ed. Pediatric Nutrition Handbook. 6th ed. Illinois: American Academy of Pediatrics, 2009; 357-386. 